\title{
Multiple wavelength Er-doped twincore fibre loop laser
}

\author{
0.Graydon, W.H.Loh, R.I.Laming and L.Dong \\ Optoelectronics Research Centre \\ University of Southampton \\ Southampton SO171BJ UK
}

\begin{abstract}
Multiwavelength laser operation of an inhomogeneously broadened Er-doped twincore fibre loop laser is demonstrated. Equal output power, separation $0.5 \mathrm{~nm}$ and linewidth $<10 \mathrm{kHz}$ was confirmed for each mode in a 3 wavelength laser. Up to 8 wavelength operation has been observed, demonstrating that twincore EDFAs can provide channel equalisation in an 8-channel multi-amplifier optical network.
\end{abstract}

\section{Introduction}

The realisation of stable, equal output power, multiple wavelength Er-doped fibre lasers at 1 $.5 \mu \mathrm{m}$ is highly attractive for use in future wavelength division multiplexing (WDM) optical communication systems. However the predominately homogeneous gain in Er-doped fibres has meant that such devices are difficult to construct as the gain is clamped by the cavity loss at only one lasing wavelength [1]. In the past to achieve stable operation, designs have either incorporated a separate gain medium for each lasing wavelength $[2,3]$ or have carefully adjusted the losses to make the effective gains equal [4,5]. The former requires many optical components whilst the latter is inherently unstable. In this paper we present a new configuration which uses a single piece of Er-doped twincore fibre to provide an inhomogeneous gain medium through macroscopic $(\mathrm{mm})$ spatial holebuming. The benefits of this approach have already been confirmed by the demonstration of twincore channel equalisers $[6,7]$. Previously such inhomogeneity has only been introduced by cooling the fibre to liquid nitrogen temperatures [8]

\section{Theory}

Twincore fibres have been studied in detail and their characteristics are well documented [9]. It is well known that two identical cores in close proximity will periodically couple light with a characteristic beatlength which is wavelength dependent. By doping both the cores with $\mathrm{Er}$, a gain medium with a degree of large scale $(\mathrm{mm})$ spatial inhomogeneity is made. The bandwidth and strength of the inhomogeneity is controlled by the twincore parameters and its length. By constructing a laser which incorporates a length of Er-doped twincore fibre, multiwavelength laser operation is possible as the available gain at each lasing wavelength is partially decoupled from the others. In addition by making the cavity unidirectional [10], single-frequency operation of each lasing line is ensured. 


\section{Experiment and Results}

The new laser design is shown in figure 1 and is a unidirectional travelling wave loop configuration consisting of a $8 \mathrm{~m}$ piece of Er-doped twincore fibre, a WDM, a circulator, a polarization controller and a multiwavelength fibre grating. The twincore was spliced to one core only at the input whilst at the output light was taken from both cores and only the even mode selected to ensure wavelength independent transmission. The twincore fibre is a $\mathrm{GeO}_{2}-\mathrm{Al}_{2} \mathrm{O}_{3}-\mathrm{SiO}_{2}$ composition with a peak absorption of $6 \mathrm{~dB} / \mathrm{m}$ at $1530 \mathrm{~nm}$ which corresponds to an Er dopant concentration of $220 \mathrm{ppm}$. It has the following characteristics, core-to-core separation $4.5 \mu \mathrm{m}$, core size $1.5 \mu \mathrm{m}$ and a core NA 0.27 , which yields a beatlength of $1.25 \mathrm{~mm}$. The multiwavelength fibre grating had peak reflectivities and bandwidths of $50 \%$ and $0.2 \mathrm{~nm}$ centred at $1543,1543.5$ and $1544.1 \mathrm{~nm}$. It was written into a boron-germania doped silica fibre by a novel phase mask technique [ 11]. The twincore fibre was pumped by $70 \mathrm{~m} \mathrm{~W}$ from a laser diode at $980 \mathrm{~nm}$ and the output was taken after the gratings with feedback suppressed by an optical isolator. The total round-trip loss in the cavity has been measured to be $13 \mathrm{~dB}$, which includes an input splice loss into the twincore of only $1 \mathrm{~dB}$.

Figure 2 shows the laser output spectra from the laser and one may clearly observe three lasing wavelengths of equal power $(390 \mu \mathrm{W})$ and resolution limited bandwidth $(0.1 \mathrm{~nm})$. To confirm single-frequency operation a HP external cavity singlemode semiconductor laser was tuned to each of the individual laser wavelengths and the beat signal was observed. A typical RF spectrum is shown in figure 3 and clearly shows that the lasing mode is a single frequency. Longitudinal mode-hopping (maximum $\pm 500 \mathrm{MHz}$ ) was observed, this is to be expected in such a long laser cavity $(20 \mathrm{~m})$ without any stabilization. This hopping could potentially be reduced by protecting the laser from environmental perturbations, shortening the cavity length and introducing a length of unpumped Er-doped fibre to write a tracking filter around the lasing mode as previously reported [ 12]. To accurately measure the average linewidth of the lasing modes a delayed self heterodyne technique was employed using $50 \mathrm{~km}$ of fibre and a linewidth of $10 \mathrm{kHz}$ was measured which is shown in figure 4.

For a comparison the twincore fibre was replaced with a single core Er-doped fibre of similar parameters. It was possible to obtain lasing at two wavelengths simultaneously by adjusting the cavity loss, however the laser was very unstable and clearly preferred to lase at just one wavelength. Simultaneous lasing at three wavelengths was not achievable.

By splicing into and out of one core of the twincore fibre it was possible to combine the inherent filtering and inhomogeneity of the twincore to obtain laser operation at eight wavelengths as shown in figure 5. Although the lasing wavelengths were less stable than when using gratings to select the lasing wavelengths, it clearly demonstrates that the principle of operation expands to many more channels. Since the operation of a ring laser is analogous to that of a long multi-amplifier link, the potential of twincore amplifiers $[6,7]$ for equalisation in multichannel WDM networks is confirmed.

\section{Summary}

Stable simultaneous lasing of three wavelengths (separation $0.5 \mathrm{~nm}$ ) at $1.5 \mu \mathrm{m}$ has been achieved using an inhomogeneously broadened Er-doped twincore fibre in a unidirectional ring laser. The wavelengths were precisely selected by a fibre grating and single-frequency operation of each ensured by the unidirectional configuration. An output power of $390 \mu \mathrm{W}$ and linewidth less than $10 \mathrm{kHz}$ was observed for each line. In addition 8 wavelength operation has been demonstrated in a similar configuration. These results confirm the potential of twincore amplifiers for automatic channel equalisation in WDM multi-amplifier links. 


\section{Acknowledgements}

O. Graydon acknowledges EPSRC for the provision of a studentship and wishes to thank M.

N. Zervas for his helpful technical discussions and C. Nash for his support.

\section{References}

[1] H. Okamura, Electron, Lett. Vol.27, No.23, pp.2155-56, 1991.

[2] J. W. Dawson et al., Appl. Phys. Lett. Vol.60, No.25, pp.3090-92, 1992.

[3] S. V. Chernikov et al., Electron. Lett. Vol.29, No. 12, pp.1089-91, 1993.

[4] N. Park et al., IEEE Photon. Tech. Lett. Vol.4, No.6, pp.540-41, 1992.

[5] H. Okamura et al., Electron. Lett. Vol.28, No.5, pp.461-63, 1992.

[6] R. I. Laming et al., Electron. Lett. Vol.29, No.6, pp.509-10, 1993.

[7] M. N. Zervas et al., J. Lightwave Tech., Vol.13, No.5, pp.721-31, 1995.

[8] E. Desurvire et al., IEEE Photon. Tech. Lett. Vol.2, No.4, pp.246, 1990.

[9] A. W. Snyder and J.D.Love, Optical waveguide theory, (Chapman and Hall), 1983.

[10] P. R. Morkel et al., Electron. Lett. Vol.26, No.10, pp.632-34, 1990.

[11] . M. J. Cole et al., submitted to conf. Photosensitivity Applications, 1995.

[12] Y. Cheng et al., Optics Lett. Vol.20, No.8, pp.875-77, 1995.

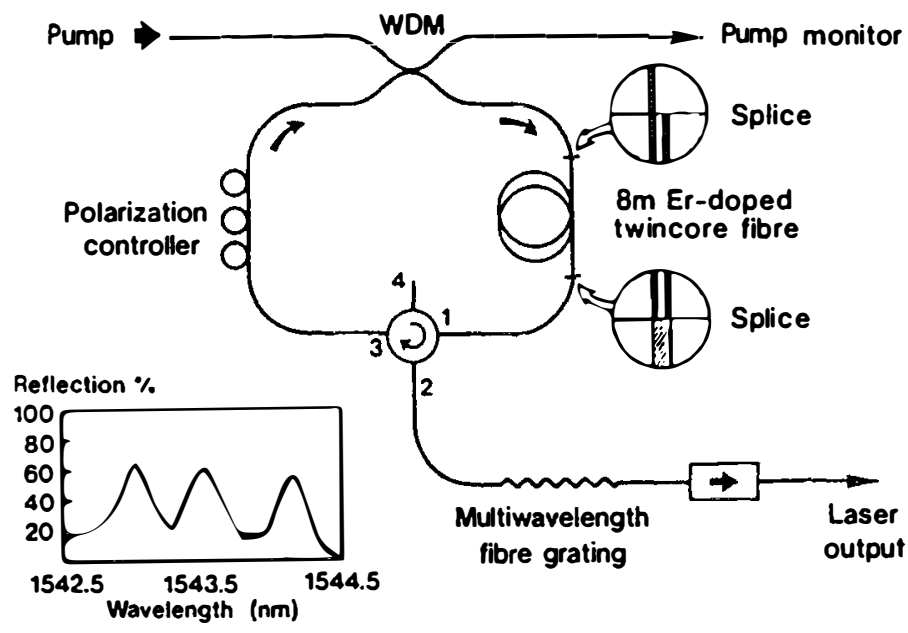

Figure 1: Schematic of the fibre laser cavity

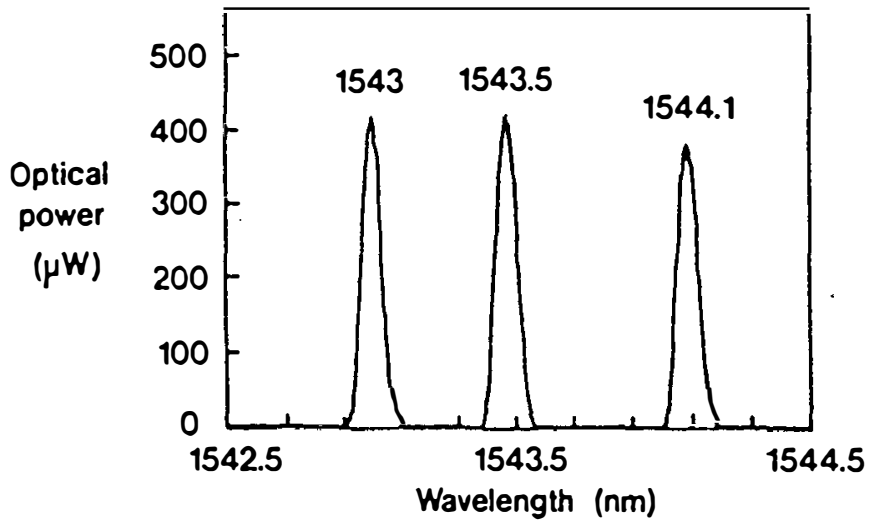

Figure 2: Laser output spectrum 


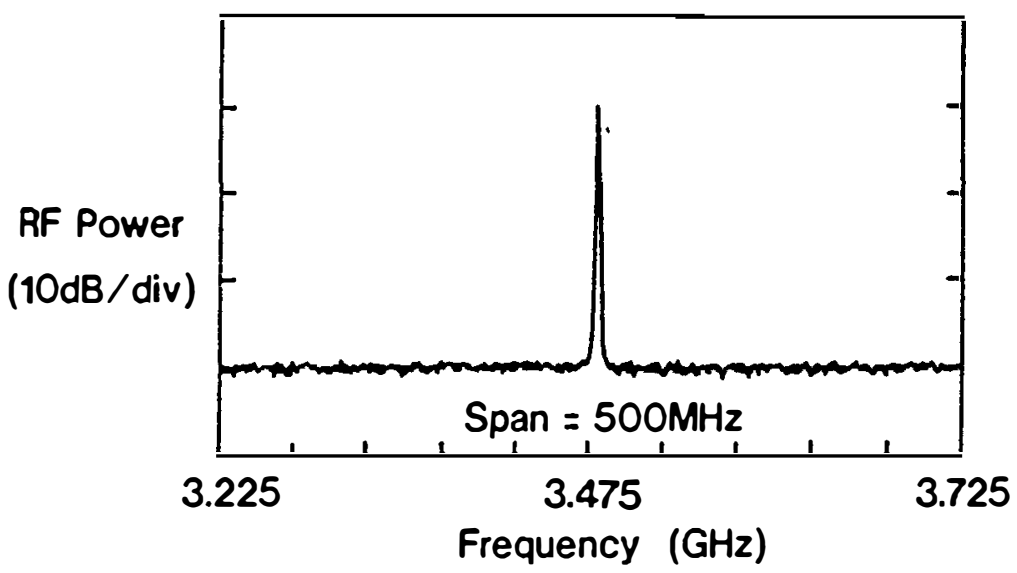

Figure 3: RF spectrum of beat frequency between one of the lasing modes and an external singlemode laser indicating single frequency operation

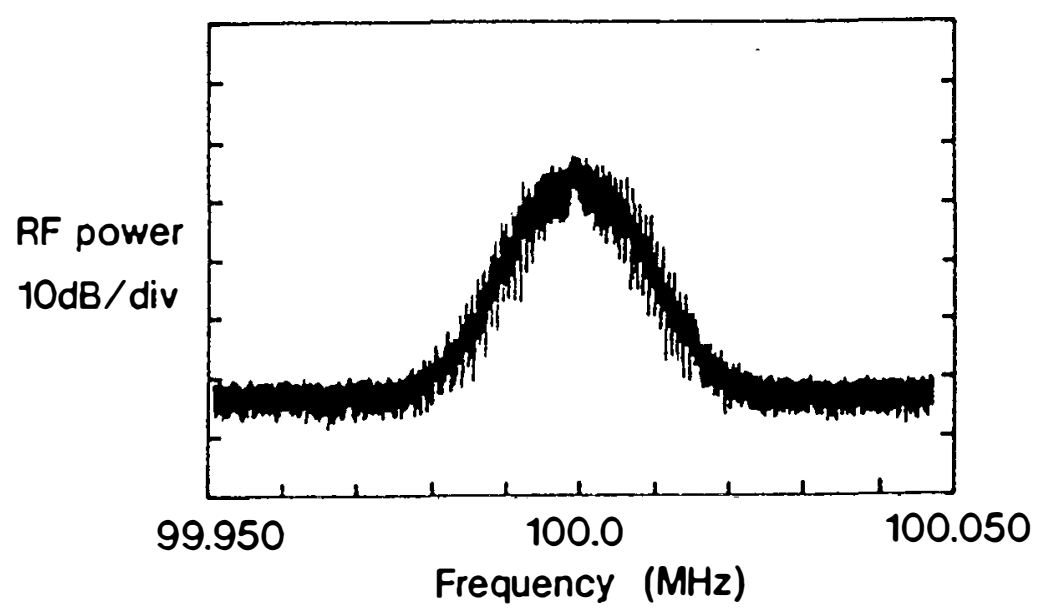

Figure 4: Self-heterodyne linewidth measurement of a single-frequency mode using a 50km delay line

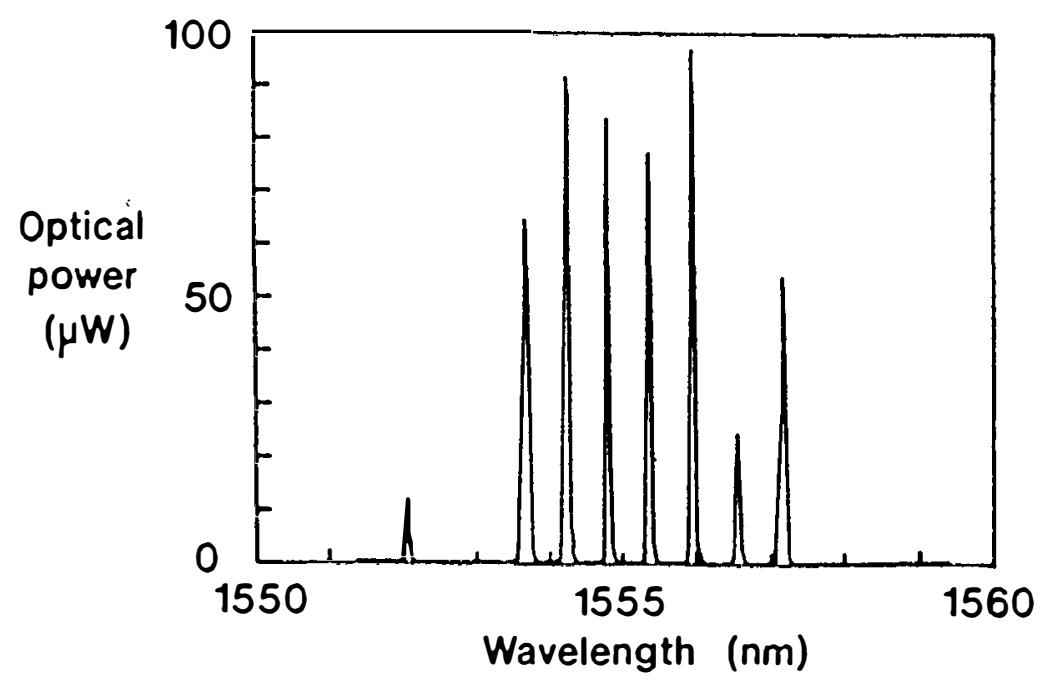

Figure 5: Eight channel operation of the Er-doped twincore fibre ring laser 